Pacific Journal of Mathematics

PRERADICALS AND INJECTIVITY 


\title{
PRERADICALS AND INJECTIVITY
}

\author{
LADISLAV BICAN
}

\begin{abstract}
Recently, the $(\rho, \sigma)$-injectivity of modules with respect to a couple of preradicals has been investigated. In the general case, the study of all the $(\rho, \sigma)$-injectivities reduces to that with $\sigma$ a torsion preradical. For a special class of rings the $(\rho, \sigma)$-injectivities are completely described. The description of all quasi-injective modules over a Dedekind domain appears as a simple corollary.
\end{abstract}

J. A. Beachy [1] has introduced a new concept of $\rho$-density of a submodule $N$ of a module $M$ and he has investigated the $(\rho, \sigma)$ injectivity of modules with respect to a couple of preradicals. In this paper we shall show that to any couple $(\rho, \sigma)$ of preradicals there exists a torsion preradical $\sigma^{\prime}$ such that the $(\rho, \sigma)$-injectivity and $\left(\rho, \sigma^{\prime}\right)$ injectivity have the same meanings. Further, in the study of $(\rho, \sigma)$ injectivity, where $\rho$ is a torsion preradical, $\rho$ can be replaced by a torsion radical. Finally, the $(\rho, \sigma)$-injectivities are completely determined for a class of subcommutative rings (containing all Dedekind domains) and this yields a characterization of all quasi-injective modules generalizing a result of Harada [7] (the methods are quite different).

We start with some basic definitions. A preradical $\rho$ for the category ${ }_{R} \mathcal{M}$ of left $R$-modules over an associative ring $R$ with unity is any subfunctor of the identity, i.e. $\rho$ assigns to each module $M$ a submodule $\rho(M)$ in such a way that every homomorphism $M \rightarrow N$ induces $\rho(M) \rightarrow \rho(N)$ by restriction. A preradical $\rho$ is said to be idempotent if $\rho^{2}=\rho$, torsion if $\rho$ is left exact and it is called a radical if $\rho(M / \rho(M))=0$. It is well-known that $\rho$ is torsion iff $L \subseteq M$ implies $\rho(L)=L \cap \rho(M)$ (see e.g. [10], Prop. 1.4). For a preradical $\rho$, a module $M$ is called $\rho$-torsion if $\rho(M)=M$ and $\rho$-torsion-free if $\rho(M)=0$. Following J. A. Beachy $[1]$ a submodule $N$ of a module $M$ is called $\rho$-dense in $M$ if $M / N \subseteq \rho(K / N)$ for some module $K$ containing $M$, or, equivalently, if $M / N \subseteq \rho(\hat{M} / N)$ where $\hat{M}$ denotes the injective hull of $M$. Finally, for a couple $(\rho, \sigma)$ of preradicals a module $Q$ is said to be $(\rho, \sigma)$-injective if for every diagram $f \downarrow Q_{Q}^{N_{0} \rightarrow N}$ with $N_{0}$ $\rho$-dense in $N$ and Ker $f \sigma$-dense in $N$ there is $g: N \rightarrow Q$ making this diagram commutative. If $\rho$ is a preradical and $M$ a module then the module $Q$ is said to be $(\rho, M)$-injective if every diagram $f \downarrow \mathcal{M}^{\mathbf{M} \rightarrow M}$ with $\boldsymbol{M}_{0}$ $\rho$-dense in $M$ can be made commutative by some homomorphism $M \rightarrow Q$. 
For a preradical $\sigma$ let $\sigma^{\prime}$ be the smallest torsion preradical which contain $\sigma$. Then $\sigma^{\prime}(M)=M \cap \sigma(\hat{M})$, so $\sigma^{\prime}(M)=\sigma(M)$ if $M$ is injective, and $M$ is $\sigma$-torsion iff 0 is $\sigma$-dense in $M$. For a preradical $\rho$ one can construct an ordinal sequence of preradicals in the following way:

$$
\begin{aligned}
\rho^{\prime}(A) & =\rho(A), \\
\rho^{\alpha+1}(A) / \rho^{\alpha}(A) & =\rho\left(A / \rho^{\alpha}(A)\right), \\
\rho^{\alpha}(A) & =\bigcup_{\beta<\alpha} \rho^{\beta}(A), \alpha \text { a limit ordinal. }
\end{aligned}
$$

As it is well-known (see [10]), the preradical $\rho^{*}$ defined by $\rho^{*}(A)=$ $\rho^{\alpha}(A)$ whenever $\rho^{\alpha}(A)=\rho^{\alpha+1}(A)$ is a radical and, in fact, the smallest radical containing $\rho$ (we put $\rho \leqq \sigma$ whenever $\rho(M) \subseteq \sigma(M)$ for all modules $\boldsymbol{M}$ ).

LeMma 1. Let $\rho, \sigma$ be preradicals for ${ }_{R} \mathcal{M}$. A module $Q$ is $(\rho, \sigma)$ injective iff it is $(\rho, M)$-injective for all modules $M$ having 0 as a $\sigma$-dense submodule.

Proof. See [1], Theorem 23.

THEOREM 2. Let $\rho, \sigma$ be preradicals for ${ }_{R} \mathcal{M}$ and let $Q \in_{R} \mathcal{M}$. Then

(a) $Q$ is $(\rho, \sigma)$-injective iff it is $\left(\rho, \sigma^{\prime}\right)$-injective,

(b) if $\rho$ is a torsion preradical, then $Q$ is $(\rho, \sigma)$-injective iff it is $\left(\rho^{*}, \sigma\right)$-injective.

Proof. For to prove (a) it suffices to use Lemma 1 , since 0 is $\sigma$-dense in $\boldsymbol{M}$ iff 0 is $\sigma^{\prime}$-dense in $M$.

If $Q$ is $\left(\rho^{*}, \sigma\right)$-injective then it is $(\rho, \sigma)$-injective since $\rho \leqq$ $\rho^{*}$. Assume that $Q$ is $(\rho, \sigma)$-injective, and let $N_{0} \subseteq N$ be $\rho^{*}$-dense, with $f: N_{0} \rightarrow Q$ and Ker $f \sigma$-dense in $N$. By a well-known argument using Zorn's lemma there exists a maximal extension $f_{1}: N_{1} \rightarrow Q$. Then $\rho\left(N / N_{1}\right)=0$, since otherwise $f_{1}$ could be extended to the $\rho$-closure of $N_{1}$ in $N$ (by (a), $\sigma$ can be assumed to be a torsion preradical), and so therefore $\rho^{*}\left(N / N_{1}\right)=\rho\left(N / N_{1}\right)=0$, which implies $N_{1}=N$.

THEOREM 3. If $R$ is left hereditary, then the following equivalent conditions hold for each preradical $\rho$ for ${ }_{R} \mathcal{M}$.

(1) $M_{0} \subseteq M$ is $\rho$-dense iff it is $\rho^{\prime}$-dense,

(2) if $M_{0} \subseteq M$ is $\rho$-dense and $M / M_{0} \cong N / N_{0}$, then $N_{0} \subseteq N$ is $\rho$-dense. 
Proof. The equivalence of conditions (1) and (2) is obvious. If $R$ is left hereditary, then if $\boldsymbol{M}_{0} \subseteq \boldsymbol{M}, \hat{\boldsymbol{M}} / \boldsymbol{M}_{0}$ is injective, and so $\boldsymbol{M}_{0}$ is $\rho$-dense in $M$ iff $M / M_{0} \subseteq \rho\left(\hat{M} / M_{0}\right)=\rho^{\prime}\left(\hat{M} / M_{0}\right)$ iff $M_{0}$ is $\rho^{\prime}$-dense in $M$.

Before proceeding we recall some basic definitions (see e.g. [2]). Let $\pi$ be the set of all pair-wise non-isomorphic simple left $\boldsymbol{R}$-modules. For every module $\boldsymbol{M}$ and every subset $\pi^{\prime} \subseteq \pi$ let us define $S_{\pi^{\prime}}(M)$ as the submodule of $M$ generated by all simple submodules of $M$ isomorphic to some module from $\pi^{\prime}$. It is easy to see that $S_{r^{\prime}}$ is a torsion preradical. The smallest radical $S_{\pi^{\prime}}^{*}$ containing $S_{\pi^{\prime}}$ (defined above) is torsion and is said to be the fundamental torsion radical. A ring $R$ is said to have primary decompositions (PD) if $S_{\pi}^{*}(M)=\sum_{U \in \pi}^{\circ} S_{U}^{*}(M)$ for every module $M$. It is well-known that for a subcommutative ring with (PD) for which $M / M^{2}$ is either 0 or a simple module for every maximal ideal $M$ there is $S_{\pi}^{*}=S_{\pi}^{\omega}$ where $\omega$ is the first infinite ordinal (see e.g. [9]). Recall ([9], Def. 7.1) that a $S_{\pi}^{*}$-torsion module $M$ is said to be quasicyclic if $S_{\pi}^{\alpha+1}(M) / S_{\pi}^{\alpha}(M)$ is either 0 or simple for all ordinals $\alpha$ and $S_{\pi}^{n}(M) \varsubsetneqq M$ for all natural integers $n$. For further purposes we denote by 0 the zero functor and by $\infty$ the identity functor. In the rest of this paper we shall deal with a subcommutative ring $R$ having (PD) such that $M / M^{2}$ is either 0 or a simple module for every maximal ideal $M$, every proper homomorphic image of $R$ is $S_{\pi}^{*}$-torsion and every preradical for ${ }_{R} \mathcal{M}$ satisfies condition (2) of Theorem 3. For easy references we shall call such a ring a BSring. The last condition is independent from all others as shows the following example: Taking as $R=Z /\left(p^{3}\right)$ the factor-ring of integers modulo $p^{3}$ and $\rho(M)=J M$ where $J$ is the Jacobson radical of $R$, we obtain a preradical which does not satisfy the condition (2) from Theorem 3, since $J \cdot C\left(p^{3}\right)=C(p)$ so that 0 is $\rho$-dense in $C\left(p^{2}\right)$. On the other hand, $C\left(p^{3}\right) / C\left(p^{2}\right)$ is not $\rho$-torsion, so that $C(p)$ is not $\rho$-dense in $C\left(p^{3}\right), C\left(p^{3}\right)$ being injective. The idempotent radical $\sigma$ on the abelian groups category assigning to each group its greatest divisible subgroup provides an example of a preradical which is not torsion and satisfies the condition (2) from Theorem 3.

THEOREM 4. Let $R$ be a BS-ring. Then $\rho \neq \infty$ is a torsion preradical for ${ }_{R} M$ iff to every $U \in \pi$ there is $n(U) \in\{N \cup\{0\} \cup\{\infty\}, N$ the set of natural integers $\}$ such that $\rho(M)=\Sigma_{U \in \pi}^{\circ} S_{U}^{n(U)}(M)$ for all $M \in{ }_{R} M$.

Proof. We can obviously restrict ourselves to the proof of the necessity. It is well known that the smallest radical $\rho^{*}$ containing $\rho$ is torsion. Now from the correspondence between torsion radicals and radical filters (see e.g. [8]) and from the fact every proper homomorphic image of $R$ is $S_{\pi}^{*}$-torsion it easily follows $\rho^{*}$ is fundamental, $\rho^{*}=S_{\pi^{\prime}}^{*}$ 
for some $\pi^{\prime} \subseteq \pi$. Thus for every $M \in_{R} M, \rho(M)=\Sigma_{U \in \pi}^{\circ} M_{U}=$ $\Sigma_{U \in \pi}^{\circ} \rho_{U}(M)$ where $\rho_{U}=S_{U}^{*} \rho$. Now it suffices to describe $\rho_{U}$. Let $I$ be a maximal ideal of $R$ such that $R / I \cong U$. Two cases can arise:

(1) All the cyclic modules $R / I^{n}, n=1,2, \cdots$ are $\rho$-torsion. If $I^{n}=I^{n+1}$ for some natural integer $n$, then $S_{U}^{n}=S_{U}^{n+1}$ and $\rho_{U}=S_{U}^{n}=$ $S_{U}^{*}$. If $I^{n} \neq I^{n+1}$ for all $n$ then as in the case of abelian groups the $U$-quasicyclic module (i.e. quasicyclic module $M$ with $\left.S_{\pi}^{n+1}(M) / S_{\pi}^{n}(M) \cong U\right)$ is a direct limit of $R / I^{n}, n=1,2, \cdots$ and thus $\rho_{U}=S_{U}^{*}$, since by [2], Theorem 3.3 every $S_{U}^{*}$ torsion module can be embedded in a direct sum of quasicyclic modules.

(2) There exists a nonnegative integer $n$ such that $R / I^{n}$ is $\rho$-torsion and $R / I^{k}, k>n$ is not $\rho$-torsion. Now by [2], Theorem 4.2 for every $M \in{ }_{R} \mathcal{M} S_{U}^{n}(M)$ is a direct sum of cyclic submodules each of which is isomorphic to some $R / I^{l}, l \leqq n$ and hence $S_{U}^{n} \leqq \rho_{U}$. On the other hand, for any $M \in_{R} \mathcal{M}$ every cyclic submodule of $\rho_{U}(M)$ is isomorphic to some $R / I^{l}, l \leqq n$, so that $I^{n} \rho_{U}(M)=0$ and $S_{U}^{n}=\rho_{U}$.

Theorem 5. Let $R$ be a BS-ring and $M$ a module. Then the following hold: injective,

(i) If $M$ is not $S_{\pi}^{*}$-torsion then a module $Q$ is $M$-injective iff it is

(ii) if $M$ is $S_{\pi}^{*}$-torsion then a module $Q$ is $M$-injective iff $S_{U}^{n(U)}(Q)=$ $S_{U}^{n(U)}(\hat{Q})$ for all $U \in \pi$, where $n(U)$ is the smallest ordinal for which $S_{U}^{n(U)}(M)=S_{U}^{n(U)+1}(M)$.

Proof. By [1], Corollary $2.9 Q$ is $M$-injective iff it is $(\infty, \rho)$ injective where $\rho$ is the smallest torsion preradical for which $M$ is torsion.

(i) Taking an element $x \in M-S_{\pi}^{*}(M)$ we have $R x \cong R, R$ being a BS-ring. Thus $\rho(R)=R$ and $\rho=\infty$. It is now obvious that $Q$ is $(\infty, \infty)$-injective iff it is injective.

(ii) By Theorem $4, \rho(N)=\sum_{U \in \pi}^{\circ} S_{U}^{n_{(U)}}(N)$ for every $N \in \in_{R} M$. As it is easily seen the numbers $n(U)$ are just the smallest ordinals for which $S_{U}^{n^{(U)}}(M)=S_{U}^{n^{(U)+1}}(M)$. By [1], Theorem 2.5 a module $Q$ is $\left(\infty, S_{U}^{n^{(U)}}\right)$-injective iff $S_{U}^{n_{U}^{(U)}}(Q)=S_{U}^{n^{(U)}}(\hat{Q})$ and the assertion follows.

Corollary 6. Let $R$ be a BS-ring and $Q$ a module. Then $Q$ is quasi-injective iff it is either injective or of the form $Q=\Sigma_{U \in \pi}^{\circ} S_{U}^{*}(Q)$ where every $S_{U}^{*}(Q), U \in \pi$ is a direct sum of pair-wise isomorphic cyclic or quasi-cyclic modules.

Proof. We proceed to the necessity, the sufficiency being obvious by Theorem 5. Suppose that $Q$ is quasi-injective. By the preceding 
Theorem $Q$ is either injective or of the form $Q=\Sigma_{U \in \pi}^{\circ} S_{U}^{*}(Q)$. By [2], Theorems 3.2 and 4.2 every $S_{U}^{*}(Q)$ is a direct sum of quasicyclic and cyclic modules, so that it suffices to use Theorem 5 (ii).

THEOREM 7. Let $R$ be a BS-ring and $\rho, \sigma \neq \infty$ be two preradicals for ${ }_{R} M$. Then there exists a module $M$ such that a module $Q$ is $(\rho, \sigma)$ injective iff it is M-injective. Moreover, $M$ can be chosen quasiinjective.

Proof. We shall divide this proof into three steps.

(1) We show that if $\rho, \sigma$ are torsion preradicals such that $\rho^{*} \cap \sigma$, $\sigma \neq \infty$ and $Q$ is a $(\infty, \rho)$-injective module, then $Q$ is $(\rho, \sigma)$ injective. So, let $Q$ be a $(\infty, \rho)$-injective module and let us consider the diagram

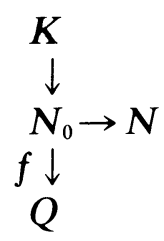

with $N / N_{0} \rho$-torsion, $N / K \sigma$-torsion, $K=\operatorname{Ker} f$. It follows from $\rho=\rho^{*} \cap \sigma$ and Theorem 4 that $\sigma(M)=\rho(M) \oplus \tau(M)$ for every $M \in_{R} M$ and a suitable torsion preradical $\tau$. For $N^{\prime} / K=\tau(N / K)$ the module $\left(N^{\prime}+N_{0}\right) / N_{0} \cong N^{\prime} / N_{0} \cap N^{\prime}$ is $\rho$-torsion and $\tau$-torsion so that $N^{\prime} \subseteq N_{0}$. Thus $f$ induces

$$
\begin{aligned}
& N_{0} / K=\tau(N / K) \oplus\left(\rho(N / K) \cap \vec{N}_{0} / K\right) \rightarrow \tau(N / K) \oplus \rho(N / K) \\
& \bar{f} \downarrow \\
& \quad Q
\end{aligned}
$$

By hypothesis, the restriction of $\bar{f}$ to $\rho(N / K) \cap N_{0} / K$ extends to a homomorphism $\rho(N / K) \rightarrow Q$ and the assertion follows easily.

(2) It follows from Theorems 2 and 3 that $Q$ is $(\rho, \sigma)$-injective iff it is $\left(\left(\rho^{\prime}\right)^{*}, \sigma^{\prime}\right)$-injective. Now by the definition $Q$ is $\left(\left(\rho^{\prime}\right)^{*}, \sigma^{\prime}\right)$-injective iff it is $\left(\left(\rho^{\prime}\right)^{*} \cap \sigma^{\prime}, \sigma^{\prime}\right)$-injective and the preceding part results that $Q$ is $(\rho, \sigma)$-injective iff it is $(\infty, \tau)$-injective, where $\tau=\left(\rho^{\prime}\right)^{*} \cap \sigma^{\prime}$.

(3) From (2) and Theorem 4 it easily follows that a module $Q$ is $(\infty, \tau)$-injective iff it is $\left(\infty, S_{U}^{n^{(U)}}\right)$-injective for all $U \in \pi$ where $\tau(M)=$

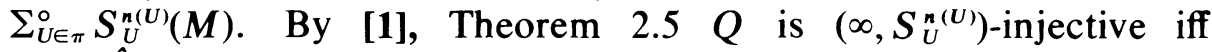
$S_{U}^{n(U)}(\hat{Q}) \subseteq Q$ and the idempotence of $S_{U}^{n^{(U)}}$ yields that $Q$ is $(\rho, \sigma)$ injective iff $S_{U}^{n(U)}(Q)=S_{U}^{n^{(U)}}(\hat{Q})$. For $U \in \pi, U \cong R / I, I$ a maximal ideal of $R$, we put $M_{U}=R / I^{n(U)}$ if $n(U) \in N \cup\{0\}$ and $M_{U}$ is an 
$U$-quasicyclic module if $n(U)=\infty$. Taking $M=\Sigma_{U \in \pi}^{\circ} M_{U}$ it suffices to use Theorem 5 (ii). $\quad M$ is quasi-injective by Corollary 6 .

Remark. M. Harada ([7], Corollary to Proposition 2.6) has described the structure of quasi-injective modules over a Dedekind domain. This description follows from our Corollary 6 immediately.

\section{REFERENCES}

1. J. A. Beachy, A generalization of injectivity, Pacific J. Math., 41 (1972), 313-328.

2. L. Bican, Kulikov's criterion for modules (to appear).

3. - QF-3' modules and rings, Comment. Math. Univ. Carol., 14 (1973), 295-303.

4. S. E. Dickson, A torsion theory for abelian categories, Trans. Amer. Math. Soc., 121 (1966), 223-235.

5. V. Dlab, A characterization of perfect rings, Pacific J. Math., 33 (1970), 79-88.

6. C. Faith, Lectures on injective modules and quotient rings, Lecture Notes in Mathematics 49, Springer Verlag, 1967.

7. M. Harada, Note on quasi-injective modules, Osaka J. Math., 2 (1965), 351-356.

8. A. P. Mišina, L. A. Skornjakov, Abelevy gruppy i moduli, Moskva 1968.

9. T. S. Shores, The structure of Loewy modules, J. reine angew. Math., 254 (1972), 204-220.

10. B. Stenström, Rings and modules of quotients, Lecture Notes in Mathematics 237, Springer Verlag, 1971.

Received September 11, 1973, and in revised form July 11, 1974.

KARLOVY UNIVERSITY 


\section{PACIFIC JOURNAL OF MATHEMATICS \\ EDITORS}

\author{
Richard ARENS (Managing Editor) \\ University of California \\ Los Angeles, California 90024
}

\author{
R. A. Beaumont \\ University of Washington \\ Seattle, Washington 98105
}

\section{J. DugundJI}

Department of Mathematics

University of Southern California

Los Angeles, California 90007

D. Gilbarg and J. Milgram

Stanford University

Stanford, California 94305

\section{ASSOCIATE EDITORS}
E. F. BECKENBACH
B. H. NeumanN
F. WOLF
K. YoshidA

\section{SUPPORTING INSTITUTIONS}

\author{
UNIVERSITY OF BRITISH COLUMBIA \\ CALIFORNIA INSTITUTE OF TECHNOLOGY \\ UNIVERSITY OF CALIFORNIA \\ MONTANA STATE UNIVERSITY \\ UNIVERSITY OF NEVADA \\ NEW MEXICO STATE UNIVERSITY \\ OREGON STATE UNIVERSITY \\ UNIVERSITY OF OREGON \\ OSAKA UNIVERSITY
}

\author{
UNIVERSITY OF SOUTHERN CALIFORNIA \\ STANFORD UNIVERSITY \\ UNIVERSITY OF TOKYO \\ UNIVERSITY OF UTAH \\ WASHINGTON STATE UNIVERSITY \\ UNIVERSITY OF WASHINGTON \\ AMERICAN MATHEMATICAL SOCIETY
}

The Supporting Institutions listed above contribute to the cost of publication of this Journal, but they are not owners or publishers and have no responsibility for its contents or policies.

Mathematical papers intended for publication in the Pacific Journal of Mathematics should be in typed form or offset-reproduced (not dittoed), double spaced with large margins. Underline Greek letters in red, German in green, and script in blue. The first paragraph or two must be capable of being used separately as a synopsis of the entire paper. Items of the bibliography should not be cited there unless absolutely necessary, in which case they must be identified by author and Journal, rather than by item number. Manuscripts, in duplicate, may be sent to any one of the four editors. Please classify according to the scheme of Math. Reviews, Index to Vol. 39. All other communications should be addressed to the managing editor, or Elaine Barth, University of California, Los Angeles, California, 90024.

100 reprints are provided free for each article, only if page charges have been substantially paid. Additional copies may be obtained at cost in multiples of 50 .

The Pacific Journal of Mathematics is issued monthly as of January 1966. Regular subscription rate: $\$ 72.00$ a year (6 Vols., 12 issues). Special rate: $\$ 36.00$ a year to individual members of supporting institutions.

Subscriptions, orders for back numbers, and changes of address should be sent to Pacific Journal of Mathematics, 103 Highland Boulevard, Berkeley, California, 94708.

PUBLISHED BY PACIFIC JOURNAL OF MATHEMATICS, A NON-PROFIT CORPORATION Printed at Jerusalem Academic Press, POB 2390, Jerusalem, Israel.

$$
\begin{gathered}
\text { Copyright (c) } 1975 \text { Pacific Journal of Mathematics } \\
\text { All Rights Reserved }
\end{gathered}
$$




\section{Pacific Journal of Mathematics}

Vol. 56, No. 2 December, 1975

Ralph Alexander, Generalized sums of distances .................. 297

Zvi Arad and George Isaac Glauberman, A characteristic subgroup of a group of odd order ............................... 305

B. Aupetit, Continuité du spectre dans les algèbres de Banach avec involution ........................................ 321

Roger W. Barnard and John Lawson Lewis, Coefficient bounds for some classes of starlike functions . ...........................

Roger W. Barnard and John Lawson Lewis, Subordination theorems for some classes of starlike fumctions . .................... 333

Ladislav Bican, Preradicals and injectivity .................. 367

James Donnell Buckholtz and Ken Shaw, Series expansions of analytic

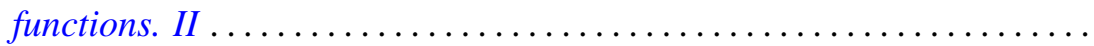

Richard D. Carmichael and E. O. Milton, Distributional boundary values in

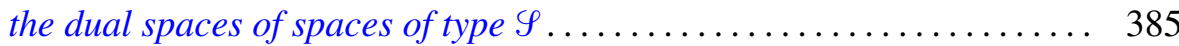

Edwin Duda, Weak-unicoherence ............................ 423

Albert Edrei, The Padé table of functions having a finite number of essential singularities ........................................

Joel N. Franklin and Solomon Wolf Golomb, A function-theoretic approach to the study of nonlinear recurring sequences ................ 455

George Isaac Glauberman, On Burnside's other $p^{a} q^{b}$ theorem 469

Arthur D. Grainger, Invariant subspaces of compact operators on topological vector spaces .........................

Jon Craig Helton, Mutual existence of sum and product integrals .

Franklin Takashi Iha, On boundary functionals and operators with finite-dimensional null spaces

Gerald J. Janusz, Generators for the Schur group of local and global number fields

A. Katsaras and Dar-Biau Liu, Integral representations of weakly compact operators.

W. J. Kim, On the first and the second conjugate points .

Charles Philip Lanski, Regularity and quotients in rings with involution ....

Ewing L. Lusk, An obstruction to extending isotopies of piecewise linear manifolds.

Saburou Saitoh, On some completenesses of the Bergman kernel and the Rudin kernel..

Stephen Jeffrey Willson, The converse to the Smith theorem for $Z_{p}$-homology spheres. 